\title{
Baseline characteristics of study sites and women enrolled in a three-arm cluster randomized controlled trial: PMTCT uptake and retention (pure) Malawi
}

Monique van Lettow ${ }^{1,2^{*}}$, Hannock Tweya ${ }^{3,4}$, Nora E. Rosenberg ${ }^{5,6}$, Clement Trapence ${ }^{3}$, Virginia Kayoyo ${ }^{5}$, Florence Kasende ${ }^{1}$, Blessings Kaunda ${ }^{7}$, Mina C. Hosseinipour ${ }^{5,8}$, Michael Eliya $^{9}$, Fabian Cataldo ${ }^{1}$, Salem Gugsa ${ }^{3}$, Sam Phiri ${ }^{3,8,10}$ and for the PURE Malawi consortium

\begin{abstract}
Background: Malawi introduced an ambitious public health program known as "Option B+" which provides all HIV-infected pregnant and breastfeeding women with lifelong combination antiretroviral therapy, regardless of WHO clinical stage or CD4 cell count. The PMTCT Uptake and REtention (PURE) study aimed at evaluating the effect of peer-support on care-seeking and retention in care.

Methods/design: PURE Malawi was a three-arm cluster randomized controlled trial that compared facility-based and community-based models of peer support to standard of care under Option B+ strategy. Each arm was expected to enroll a minimum of 360 women with a total minimum sample size of 1080 participants. 21 sites (clusters) were selected for inclusion in the study. This paper describes the site selection, recruitment, enrollment process and baseline characteristics of study sites and women enrolled in the trial.
\end{abstract}

Results: Study implementation was managed by 3 partner organizations; each responsible for 7 study sites. The trial was conducted in the South East, South West, and Central West zones of Malawi, the zones where the implementing partners operate. Study sites included 2 district hospitals, 2 mission hospitals, 2 rural hospitals, 13 health centers and 1 private clinic. Enrollment occurred from November 2013 to November 2014, over a median period of 31 weeks (range 17-51) by site. A total of 1269 HIV-infected pregnant (1094) and breastfeeding (175) women, who were eligible to initiate ART under Option B+, were enrolled. Each site reached or surpassed the minimum sample size. Comparing the number of women enrolled versus antenatal cohort reports, sites recruited a median of 90\% (IQR 75-100) of eligible reported women. In the majority of sites the ratio of pregnant and lactating women enrolled in the study was similar to the ratio of reported pregnant and lactating women starting ART in the same sites. The median age of all women was 27 (IQR 22-31) years. All women have $\geq 20$ months of possible follow-up time; $96 \% \geq 2$ years (24-32 months).

Conclusion: The PURE Malawi study showed that 3 implementing partner organizations could successfully recruit a complex cohort of pregnant and lactating women across 3 geographical zones in Malawi within a reasonable timeline.

Trial registration: This study is registered at clinicaltrials.gov - ID Number NCT02005835. Registered 4 December, 2013.

Keywords: PMTCT, Uptake, Retention, Peer-support, Cluster randomized trial, Malawi, Feasibility

\footnotetext{
*Correspondence: m.vanlettow@dignitasinternational.org

${ }^{1}$ Dignitas International, Zomba, Malawi

2Dalla Lana School of Public Health, University of Toronto, Toronto, Canada

Full list of author information is available at the end of the article
} 


\section{Plain english summary}

Malawi introduced a new strategy that aims at preventing HIV infection from mother to child. There is no cure for HIV, but medication (antiretroviral treatment) is available to slow the progression of the virus and help prevent transmission of HIV to others. The so called "Option $\mathrm{B}+$ " strategy means that all HIV-infected pregnant and breastfeeding women start antiretroviral treatment as soon as they are diagnosed. The PMTCT Uptake and REtention (PURE) study evaluated whether support provided by peers (mothers who are HIVinfected and on treatment themselves) can help to increase the number of HIV-infected pregnant and breastfeeding women to start treatment (uptake) and keep them in care and on treatment (retention). The study took place in 21 health facilities, where mothers either received no peer support (7 sites), peer support in the facility ( 7 sites) or peer support in the community (7 sites). This article describes how the study was successfully set up and managed by 3 organizations. Between November 2013 and November 2014, a total of 1269 HIVinfected women were included in the study in the 21 health facilities. We describe that it is possible to set up a complex study across 3 geographical zones in Malawi.

\section{Background}

In 2011, Malawi introduced an ambitious public health strategy known as "Option B+", providing a standardized lifelong combination antiretroviral therapy (ART) regimen to all HIV-infected pregnant and breastfeeding women, irrespective of their CD4 cell count or World Health Organisation (WHO) clinical stage of HIV infection. This public health approach for the prevention of mother to child transmission (PMTCT) of HIV has since been adopted by several other countries in the region and was included in the updated WHO guidelines in 2013 [1]. Initial data have demonstrated that the Option $\mathrm{B}+$ strategy has resulted in large increases in the number of pregnant women accessing PMTCT [2]. However, concerns have been raised about the level of attrition of women from care [3]. Although the strategy offers an attractive rapid ART scale-up and has the potential to profoundly impact maternal and infant outcomes, services supporting the implementation of Option B + have not been formally evaluated. Several issues were identified at the national level as potential threats to the successful implementation and scale up of the strategy: a) potential suboptimal uptake of ART by asymptomatic pregnant women due to low treatment literacy and stigma; b) low adherence to ART and poor follow-up of HIV exposed infants; and c) lack of psycho-social support for long term retention in this relatively asymptomatic patient population [4]. Hence, it seemed apparent that the identification of interventions to support PMTCT uptake and retention would be the first step towards improvement. A recently published systematic review identified 9 completed studies and 5 ongoing trials which examined initiation of ART in pregnant women [5]. While the authors report several promising interventions for improving ART initiation, the quality of evidence was insufficient to support recommendations. In addition, results for ART initiation in pregnant women were not independently examined, and maternal retention in PMTCT care and exposed infants care were not assessed.

Although randomized controlled trials are promoted as the highest level of evidence for the evaluation of interventions, the implementation of rigorous trials within the context of the public health sector are complex and therefore still uncommon [6]. PURE Malawi embraced the challenge of implementing a multi-site controlled trial within the context of public health clinics under real world conditions in Malawi. PURE Malawi was a three-arm cluster randomized controlled trial that aimed at evaluating whether facility- or community-based peersupport would enhance care-seeking and retention in care by HIV-infected pregnant and breastfeeding women, their HIV-exposed infants, and their male sex partners, and ultimately improve health outcomes in all three populations. The hypothesis was that enhanced support for women and their families within facilities and/or community would result in improved retention in the continuum of PMTCT care. The primary trial outcome is the proportion of women retained in care, 2 years after they initiated antiretroviral therapy under the Option B+ strategy. This paper describes the site selection, recruitment, enrollment process and baseline characteristics of study sites and women enrolled in the trial.

\section{Methods}

PURE Malawi was a three-arm stratified cluster randomized controlled trial that compared facility-based and community-based models of peer support to standard of care under Option $\mathrm{B}+$. The three arms differed with respect to the presence and location of one-on-one patient education and support, support groups, visit reminders, and missed visit follow-up. The trial was unblinded to study participants and health care providers, but blinded to outcome assessors. A comprehensive description of the study arms was reported elsewhere [7]. The PURE trial was managed by the PURE Malawi consortium comprising of governmental, non-governmental, research, and academic partners. The consortium was led by Lighthouse Trust. Dignitas International, The University of Malawi College of Medicine Malaria Alert Centre, and University of North Carolina Project were each responsible for trial implementation in one Malawian zone. The trial was conducted in the South East, South West, and Central West zones of Malawi, the zones where the implementing 
partners operate. Selection and responsibilities of all consortium members were described elsewhere [7].

\section{Site selection and recruitment targets}

Clusters (i.e. health facilities) were the unit of randomization in this trial. Public health facilities in the South East, South West, and Central West health zones of Malawi, covering the central and southern geographical regions of Malawi, were assessed for eligibility in the trial. Sites were eligible if they provided Option B+ services, did not have additional PMTCT interventions or research activities beyond the Malawi national standard of care, and were expected to have at least 20 eligible women for Option B+ in a 6 month period. HIV care in Malawi is free and standardized in all public health facilities. Site assessments, selection and randomization of the sites were described elsewhere [7].

Twenty-one selected sites were stratified into 3 groups of 7, with each group having sites of comparable size and expected retention, based on Ministry of Health historic data. Depending on the size of the health facility, each site was expected to reach a minimum enrollment target of $20,50,75$, or 125 pregnant or breastfeeding women eligible for the Option $\mathrm{B}+$ program. Each arm was expected to enroll a minimum of 360 women with a total minimum sample size of 1080 participants. Sites could exceed this target if they finished enrollment prior to a 6 month period.

\section{Enrolment process and index participants}

Prior to study implementation, all Ministry of Health staff working at ANC and ART departments in the selected sites received training in study arm specific standard operating procedures, adherence to ethical standards and management of data collection tools (Good Clinical Practice). At each site, a PURE site coordinator was selected to oversee the study implementation.

All newly identified HIV positive women identified during pregnancy, delivery, or breastfeeding while seeking antenatal care (ANC) or initiating ART services at the study sites were eligible for inclusion in the study. Women consented to participate and be followed in the site where they had sought ANC or ART services and were explained in which arm the site was allocated.

Based on existing PMTCT monitoring data, we anticipated 6 months until sample size was achieved.

All participating women were assigned a unique study ID. Study ID stickers were placed in the study register, the mother's existing health passport and on all routine clinic records for data extraction and tracking.

The index participants were newly confirmed HIVinfected pregnant and breastfeeding women; eligible to initiate ART under Option B+, in the 21 selected health facilities. To attain the primary trial outcome, enrolled women were expected to be followed until the end of the study period (31st July 2016), transfer out, default, or death.

\section{Data collection and management during recruitment period}

All newly identified HIV positive women were eligible for inclusion in the study. Therefore, routinely collected health facility cohort data prepared during quarterly supervision visits [8] was used to compare the number of women enrolled in the study with the number of eligible women seeking care at the health facility during the recruitment period. Monthly ANC cohort data included the number of women newly registered, newly diagnosed with HIV or already known HIV-infected, and those already on ART. ART cohort data included the number of pregnant and lactating women having started ART per quarter.

Facility characteristics data, including availability of HIV Testing Services (HTS), ANC and ART services were collected by existing study personnel throughout the enrolment period.

During study enrollment, health facility staff used PURE registration and PURE enrolment forms to collect data on mothers' age, expected delivery date (pregnant women) or date of birth of the child (lactating women), marital status, and whether the partner lives in the catchment area.

At the ART initiation visit, a routinely used clinical card was initiated and treatment initiation data were recorded, including date, age and reason for initiation of ART (WHO stage or pregnant/lactating), TB and KS. The data collection and analytic methods, including a detailed analysis plan, for the final trial outcomes were described elsewhere [7].

\section{Compensation of health facility for study specific activities}

During the enrolment period, each health facility received 1000 MK (equivalent to approximately 2 USD) per correctly enrolled study participant, in addition to a median amount of 5000 MK per month (equivalent to approximately 10 USD) for correct organization and storage of clinical and study records as stipulated by the national guidelines and PURE protocols. This amount was shared among the facility staff involved in the study.

\section{Results}

PURE Malawi sites included 2 district hospitals, 2 mission hospitals, 2 rural hospitals, 13 health centers and 1 private clinic. Table 1 shows PURE Malawi sites by health zone, implementing partner and study arm. Each implementing partner managed the implementation of the study across the 7 sites in the respective health zone where the partners operate. The organization Mothers2Mothers provided 
Table 1 PURE Malawi sites by health zone, implementing partner and study arm

\begin{tabular}{|c|c|c|c|}
\hline \multirow{2}{*}{$\begin{array}{l}\text { Implementing } \\
\text { partner }\end{array}$} & Central west zone & \multirow{2}{*}{$\begin{array}{l}\text { South east zone } \\
\text { Dignitas International, Zomba }\end{array}$} & \multirow{2}{*}{$\begin{array}{l}\text { South west zone } \\
\text { Malaria Alert Centre, Blantyre }\end{array}$} \\
\hline & University of North Carolina Project, Lilongwe & & \\
\hline \multirow[t]{3}{*}{ Arm 1} & Daeyang Luke Hospital Public ${ }^{c}$ & Mulanje District Hospital $^{a}$ & Mwanza District Hospital $^{a}$ \\
\hline & Kasinje Health Centre ${ }^{d}$ & Mimosa Dispensary ${ }^{\mathrm{e}}$ & Chirimba Health Centre $^{d}$ \\
\hline & Dr David Livingstone Memorial Clinic $^{f}$ & & \\
\hline \multirow[t]{4}{*}{ Arm 2} & Lobi Rural Hospital ${ }^{\mathrm{C}}$ & Muloza Health Centre $^{d}$ & Makhetha Health Centre ${ }^{d}$ \\
\hline & & Mbiza Health Centre ${ }^{d}$ & Masenjere Health Centre ${ }^{d}$ \\
\hline & & & Mdeka Health Centre ${ }^{d}$ \\
\hline & & & Kadidi Health Center ${ }^{d}$ \\
\hline \multirow[t]{3}{*}{ Arm 3} & Biriwiri Health Centre ${ }^{d}$ & Mulanje Mission Hospital $^{b}$ & Trinity Mission Hospital ${ }^{b}$ \\
\hline & Nsipe Health Centre ${ }^{d}$ & Mulomba Health Centre ${ }^{d}$ & \\
\hline & Bilira Health Center $^{d}$ & Mpala Health Centre ${ }^{d}$ & \\
\hline
\end{tabular}

${ }^{\text {a District Hospital }}$

${ }^{\mathrm{b}}$ Mission Hospital

${ }^{c}$ Rural Hospital

dHealth Centre

eDispensary

fPrivate clinic

additional support for training and implementation in all Arm 2 sites. Table 2 shows the number of Ministry of Health staff trained by site and cadre prior to study implementation. Enrollment initiation varied per trial site from November 2013 through January 2014. Recruitment was completed in November 2014. Median enrollment time per site was 31 weeks, ranging from 17 to 51 weeks. Table 3 describes the number of pregnant and lactating women enrolled per site and arm, in addition to the number of eligible women reported in cohort reports at ANC and ART during the recruitment period. Each site and arm reached or surpassed the minimum sample. However, a few participants (4\%) were enrolled later than the desired end date of July 31st 2014, making 2 years of follow up impossible by July 31,2016 , the trial end date.

A total of 1269 women were enrolled, 1094 (86\%) pregnant and 175 (14\%) lactating women.

When comparing number of enrolled pregnant women per site with facility cohort data; 7 sites recruited $>100 \%$ of the total reported cohort of HIV-infected pregnant women at ANC in the corresponding months. The additional number of pregnant women may have started ART, but not attended ANC in the specific study site. Nine sites recruited $>90 \%, 10$ sites $60-90 \%$, and 2 sites $40 \%-59 \%$ of the total reported cohort of pregnant women at ANC in the corresponding months. When comparing the number of women enrolled versus ANC reports, sites recruited a median of $90 \%$ (IQR 75-100) of eligible reported women from ANC.

Following quarterly ART cohort report data; in all study sites, a total of 1934 (81\%) pregnant and 454 (19\%) lactating women had started ART during corresponding quarters. This proportion of pregnant and lactating women was comparable to our study sample.

\section{Baseline characteristics of study sites}

Table 4 shows the characteristic of study sites, with regards to services available during the enrolment period. During the enrolment period, 9 facilities experienced a median of 2 weeks of HIV test kit stock outs (range 2-8) and 13 facilities were not able to provide HTC for a median of 4 weeks (range 1-8) due to stock outs or unavailability of HTC providers. The 6 largest facilities provided ANC and ART services during all week days; in all other facilities ANC and ART services were provided at a median of 3 days (range 2-4) and 1 day (range 1-4) per week, respectively. In 19 of the 21 facilities, ART provision was available at ANC.

\section{Baseline characteristics of women enrolled in the trial}

Cumulative baseline characteristics of women enrolled in the trial are shown in Table 5.

The median age of all women was 27 (IQR 22-31) years, with no difference between those pregnant and lactating. Pregnant women were enrolled at a median of 4 months (IQR 2-5) prenatal; breastfeeding women at a median of 5 months (IQR 1-11) postnatal. Reason for ART initiation for all women was being pregnant or lactating (regardless of CD4 or WHO stage). Among those with WHO stage reported at ART initiation, $98 \%$ of both pregnant and lactating women were reported clinically asymptomatic. There was 1 documented TB case (within the last 2 years) and 1 documented KS case at ART initiation.

Proportionally more lactating women reported being divorced, widowed, or having no sexual partner than pregnant women. Among women with at least one sexual partner, $11 \%$ were reported not living in the same catchment area as their partner. There was no difference in recruitment by Health Zone period or the time for 
Table 2 Number of facility staff trained in study procedures and good clinical practices

\begin{tabular}{|c|c|c|c|c|c|}
\hline Arm and facility name & ART/PMTC coordinators & ART/PMTCT providers & HTC providers & ART clerks & Total \\
\hline \multicolumn{6}{|l|}{ Arm 1: Standard } \\
\hline Mulanje District Hospital & 3 & 7 & 3 & 2 & 15 \\
\hline Daeyang Luke Hospital Public & 2 & 7 & 3 & 1 & 13 \\
\hline Mwanza District Hospital & & 1 & & 2 & 3 \\
\hline Mimosa Dispensary & & 2 & 2 & 2 & 6 \\
\hline Kasinje Health Centre & & 4 & 2 & 2 & 8 \\
\hline Dr. David Livingstone Memorial Clinic & & 6 & & 1 & 7 \\
\hline Chirimba Health Centre & 2 & 6 & & 2 & 8 \\
\hline \multicolumn{6}{|l|}{ Arm 2: Facility Support } \\
\hline Muloza Health Centre & & 3 & 4 & 2 & 9 \\
\hline Makhetha Health Centre & 1 & 13 & 1 & & 15 \\
\hline Mbiza Health Centre & 1 & 4 & 2 & 2 & 9 \\
\hline Masenjere Health Centre & & & 2 & 4 & 6 \\
\hline Mdeka Health Centre & 4 & 6 & 2 & 2 & 14 \\
\hline Lobi Rural Hospital & & 3 & 2 & & 5 \\
\hline Kadidi Health Centre & & 7 & & & 7 \\
\hline \multicolumn{6}{|l|}{ Arm 3: Community Support } \\
\hline Mulanje Mission Hospital & 1 & 8 & 3 & 2 & 14 \\
\hline Trinity Mission Hospital & & & 5 & 10 & 15 \\
\hline Mulomba Health Centre & & 2 & 4 & 1 & 7 \\
\hline Mpala health Centre & 1 & 3 & 2 & 2 & 8 \\
\hline Biriwiri Health Centre & & 3 & 3 & 2 & 8 \\
\hline Nsipe Health Centre & & 4 & 2 & 1 & 7 \\
\hline Bilira Health Center & & 3 & 2 & 2 & 7 \\
\hline
\end{tabular}

follow-up between pregnant and lactating women. The largest proportion of women was recruited between January and July 2014, enabling 96\% of women to be followed up for at least 2 years (24-32 months) as part of the trial.

\section{Discussion}

This paper shows that the PURE consortium was successful in recruiting a complex cohort of pregnant and lactating women across 3 geographical zones in Malawi. Each site and trial arm reached or surpassed the minimum required sample size, and the majority of sites recruited over $90 \%$ of the reported eligible women attending ANC during the recruitment period. Although cluster designs are often avoided due to the challenges of enrolling at multiple sites within a reasonable timeline, we show that, despite the absence of remunerated study staff within study site, only few participants were enrolled later than the desired end date; allowing for $96 \%$ of the cohort to be followed for at least 2 years. We report that there were HIV test kit stock outs or unavailability of the HTC providers, which may have prolonged the enrolment period. Shortages and stock out of commodities at health facilities is a reality in Malawi, however, national guidelines are set up to improve the supply management [8]. In the majority of participating sites, the ratio of pregnant and lactating women enrolled in the study was similar to the ratio of pregnant and lactating women initiated on ART in the same sites. There was no difference in age between the enrolled pregnant and lactating women. One fifth of the women enrolled were young mothers (15-21), which is consistent with national evaluations that describe women in Malawi's Option B+ program [3]. Proportionally more lactating women reported being divorced, widowed or having no sexual partner. As male partner support is thought to be an important determinant for uptake and outcomes of PMTCT initiatives [9-11], follow up of our cohort of mothers with and without partners will add to the evidence on the impact of male partner involvement approaches on PMTCT services. According to Malawi's demographic health survey, $14 \%$ of Malawian women are married to a man with more than one wife. Polygamy is most common in the Northern Region [12], which may explain why we found a lower proportion of women in polygamous marriage (9\%). In 


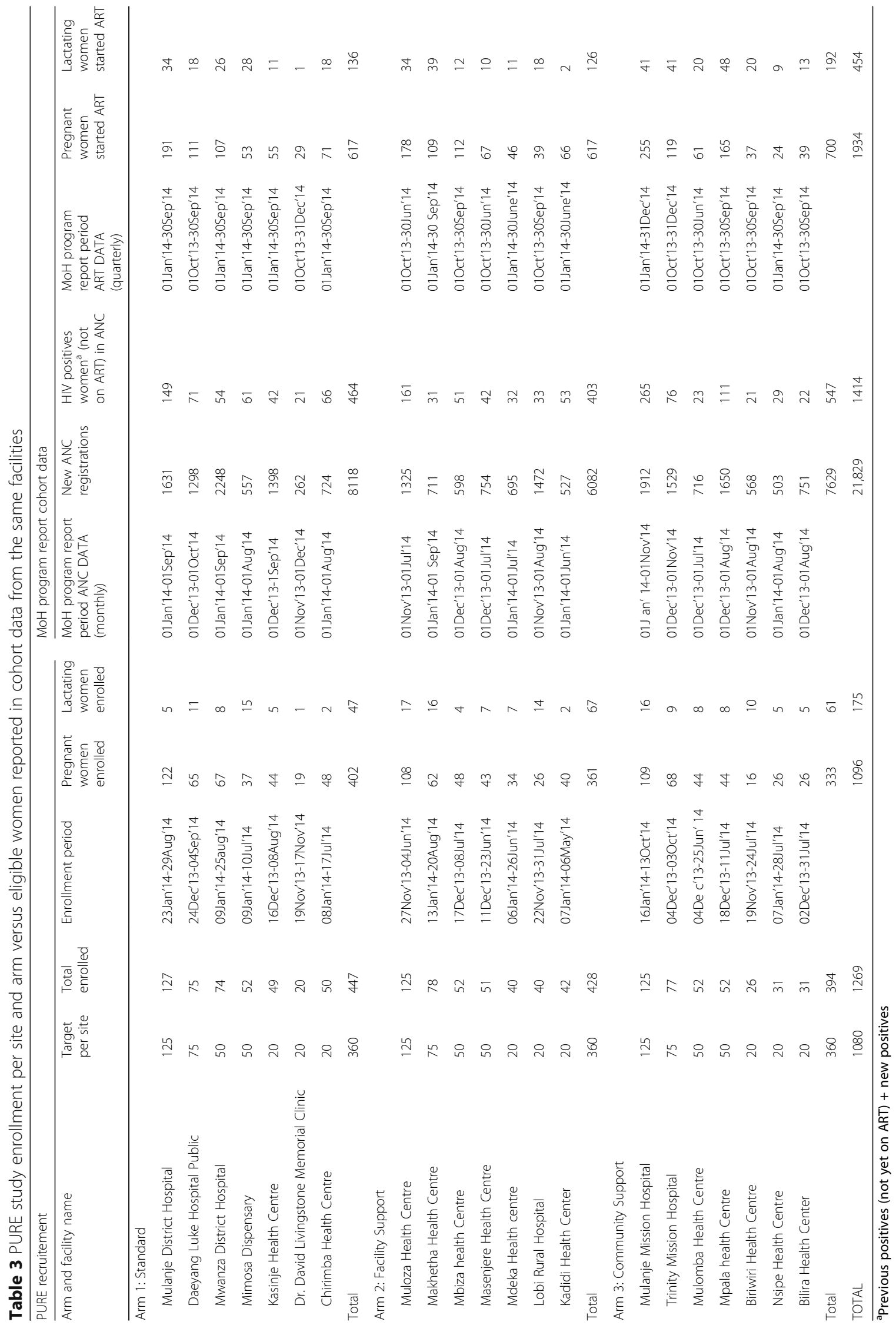


Table 4 Facility characteristics during enrollment period

\begin{tabular}{|c|c|c|c|c|c|c|}
\hline Arm and facility name & $\begin{array}{l}\text { Enrolment } \\
\text { duration } \\
\text { in weeks }\end{array}$ & $\begin{array}{l}\text { HIV test kit } \\
\text { stockout in } \\
\text { weeks }\end{array}$ & $\begin{array}{l}\text { HTC service } \\
\text { NOT available } \\
\text { in weeks }\end{array}$ & $\begin{array}{l}\text { Number of days } \\
\text { per week ANC } \\
\text { provided }\end{array}$ & $\begin{array}{l}\text { Number of days } \\
\text { per week ART } \\
\text { provided }\end{array}$ & $\begin{array}{l}\text { ART provided } \\
\text { at ANC (Yes/No) }\end{array}$ \\
\hline \multicolumn{7}{|l|}{ Arm 1: Standard } \\
\hline Mulanje District Hospital & 31 & - & - & 5 & 5 & Yes \\
\hline Daeyang Luke Hospital Public & 36 & - & - & 4 & 5 & Yes \\
\hline Mwanza District Hospital & 33 & 2 & 2 & 5 & 5 & Yes \\
\hline Mimosa Dispensary & 26 & - & 4 & 2 & 1 & Yes \\
\hline Kasinje Health Centre & 33 & - & 1 & 5 & 4 & Yes \\
\hline Dr. David Livingstone Memorial Clinic & 51 & - & 4 & 5 & 5 & No \\
\hline Chirimba Health Centre & 27 & - & - & 4 & 1 & Yes \\
\hline \multicolumn{7}{|l|}{ Arm 2: Facility Support } \\
\hline Muloza Health Centre & 27 & 4 & 4 & 3 & 1 & Yes \\
\hline Makhetha Health Centre & 31 & 8 & 8 & 3 & 1 & No \\
\hline Mbiza health Centre & 29 & - & - & 2 & 1 & Yes \\
\hline Masenjere Health Centre & 27 & 8 & 8 & 2 & 2 & Yes \\
\hline Mdeka Health centre & 24 & 2 & 2 & 2 & 1 & Yes \\
\hline Lobi Rural Hospital & 36 & - & - & 5 & 5 & Yes \\
\hline Kadidi Health Center & 17 & 2 & 2 & 2 & 1 & Yes \\
\hline \multicolumn{7}{|l|}{ Arm 3: Community Support } \\
\hline Mulanje Mission Hospital & 38 & - & - & 5 & 5 & Yes \\
\hline Trinity Mission Hospital & 43 & 4 & 4 & 5 & 5 & Yes \\
\hline Mulomba Health Centre & 29 & - & 4 & 2 & 1 & Yes \\
\hline Mpala health Centre & 29 & - & - & 3 & 1 & Yes \\
\hline Biriwiri Health Centre & 35 & 2 & 3 & 3 & 1 & Yes \\
\hline Nsipe Health Centre & 29 & - & - & 3 & 1 & Yes \\
\hline Bilira Health Center & 34 & 2 & 2 & 3 & 1 & Yes \\
\hline
\end{tabular}

our study, 98\% of pregnant and lactating women were reported clinically asymptomatic. This high proportion of women starting ART in the earliest stages is an indication of the success of Malawi's PMTCT programming.

Malawi, as the first country to implement Option $\mathrm{B}+$, has the potential to be a leader in developing and documenting highly effective models of service delivery to pregnant and lactating women and enhance retention in lifelong care and treatment. Cluster randomized controlled trials are needed to determine whether promising interventions are indeed effective. However, the implementation of such trials pose a substantial challenge, especially across multiple regions, different facility types, and several implementing partners. Thus, the ability to select 21 comparable clinics, stratify them in a meaningful way, and have each enroll its target sample size is an achievement. This achievement stems from clear leadership, small incentives to clinical staff, and wellcoordinated teams.

This paper described the processes for site selection, recruitment, and enrollment of study participants, along with baseline characteristics of study sites and women enrolled in the trial. Follow-up of participants was completed on July 31st, 2016. The final results and outcomes (per protocol) of this study are reported elsewhere [13]. Alongside this trial, a qualitative study has been implemented to explore experiences of patients and health care workers in relation to the implementation of Option $\mathrm{B}+$ and the trial interventions [14].

\section{Limitations}

We were not able to report the precise number of women who refused to participate in the study, as this data was not collected consistently across the sites. However, compared to facility cohort data, it appears that we were able to enroll the majority of eligible women.

\section{Conclusions}

Malawi, as the first country to implement Option $\mathrm{B}+$, has the potential to be a leader in developing and documenting highly effective models of service delivery to 
van Lettow et al. Reproductive Health (2017) 14:82

Page 8 of 9

Table 5 Cumulative Baseline characteristics of women included in the study

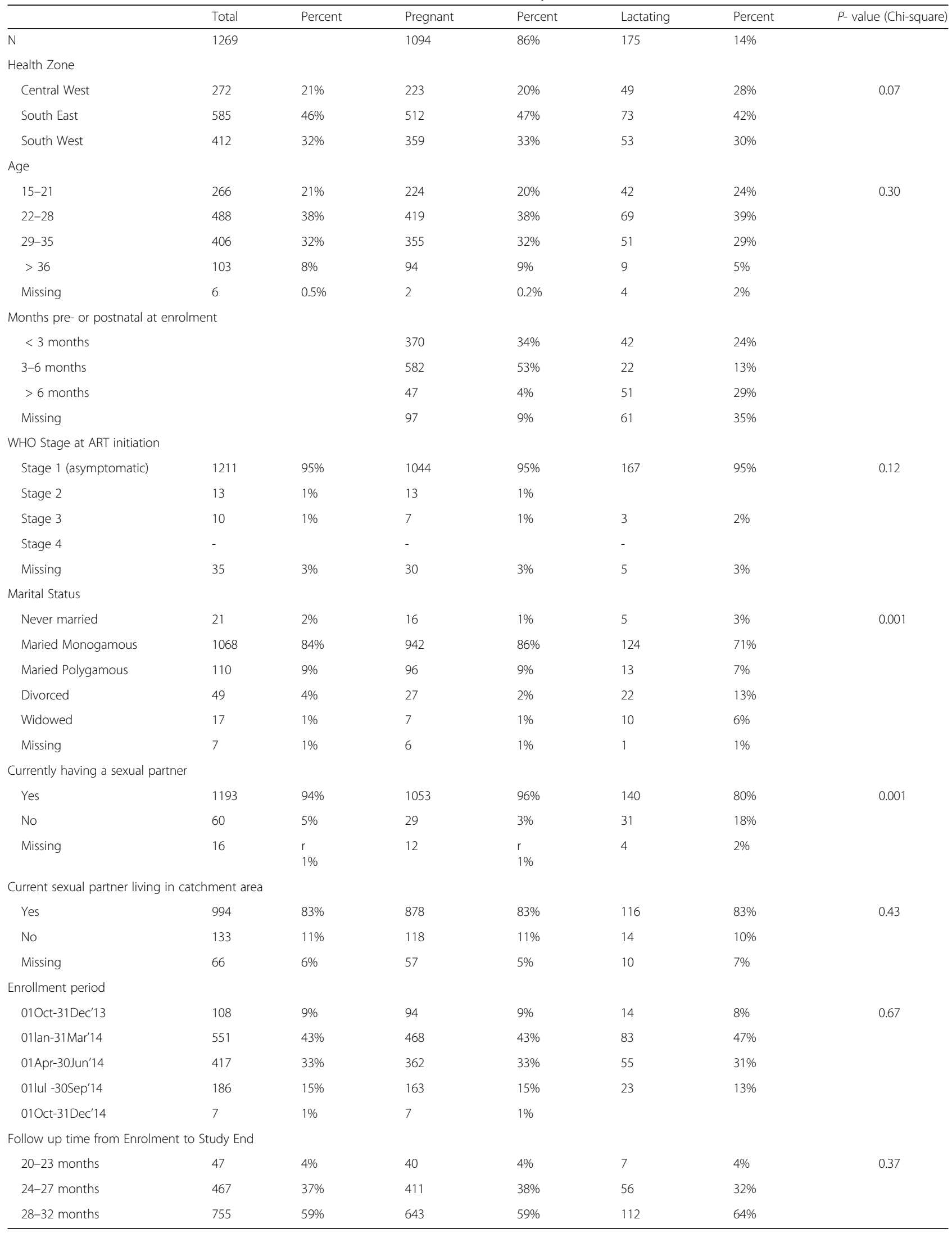


provide needed support to women and enhance retention in lifelong HIV care and treatment approaches. The PURE trial is expected to play a critical role in this process. We show that a consortium of organizations was successful in recruiting a complex cohort of pregnant and lactating women across 3 geographical zones in Malawi within a reasonable timeline.

\section{Acknowledgements}

We wish to thank the support of all members of the PURE Malawi Consortium

PURE Malawi Consortium

Sam Phiri, Hannock Tweya, Salem Gugsa, Clement Trapence, Saulos Mhlanga (Lighthouse Trust, Lilongwe, Malawi); Frank Chimbwandira, Michael Eliya (Ministry of Health, Lilongwe, Malawi); Mina Hosseinipour, Nora E. Rosenberg, Innocent Mofolo, Virginia Kayoyo, Christopher Stanley (University of North Carolina Project, Lilongwe, Malawi); Monique van Lettow, Fabian Cataldo, Misheck Nkhata, Florence Kasende, Megan Landes, (Dignitas International, Zomba, Malawi); Don Mathanga, Atupele Kapito-Tembo, Blessings Kaunda-Khangamwa (University of Malawi, College of Medicine, Malaria Alert Centre, Blantyre, Malawi); Levison Chiwaula, Gowokani Chirwa (University of Malawi, Chancellor College, Zomba, Malawi); Erik Schouten (Management Sciences for Health, Lilongwe, Malawi); Veena Sampathkumar (Mothers2Mothers, Lilongwe, Malawi).

\section{Funding}

The study was funded by the World Health Organization through an award for the INtegrating and Scaling up PMTCT through Implementation REsearch (INSPIRE) initiative from Global Affairs Canada and AIDS fonds that provided support for virologic and resistance assessment.

\section{Availability of data and materials}

The datasets used and analyzed during the current study available from the corresponding author on reasonable request.

\section{Disclaimers (WHO/GAC)}

The opinions expressed in this article do not necessarily reflect the views of policies of the World Health Organization, Global Affairs Canada or AIDS fonds.

\section{Authors' contributions}

$M v L, H T, N E R, M C H, M E, F C$ and SP contributed to the concept and design of the study. CT, VK, FK, and BK contributed to acquisition of data. MVL, HT and NER contributed to data analysis and/or interpretation of the data. MvL drafted the first manuscript. NER, MVH, FC, SG and SP reviewed and suggested revisions. All authors reviewed and approved the final manuscript.

\section{Ethics approval and consent to participate}

Ethical approval was granted through Malawi's National Health Sciences Research Committee, the University of North Carolina Institutional Review Board, the University of Toronto, and the Ethics Review Committee of the World Health Organization. All women provided written informed consent. There were no protocol amendments.

\section{Consent for publication}

Not applicable.

\section{Competing interests}

The authors declare that they have no competing interests.

\section{Publisher's Note}

Springer Nature remains neutral with regard to jurisdictional claims in published maps and institutional affiliations.

\section{Author details}

'Dignitas International, Zomba, Malawi. ${ }^{2}$ Dalla Lana School of Public Health, University of Toronto, Toronto, Canada. ${ }^{3}$ Lighthouse Trust, Lilongwe, Malawi. ${ }^{4}$ The International Union against Tuberculosis and Lung Disease, Paris, France. ${ }^{5}$ University of North Carolina Project, Lilongwe, Malawi. ${ }^{6}$ Department of Epidemiology, University of North Carolina, Chapel Hill, NC, USA.
7University of Malawi, College of Medicine, Malaria Alert Centre, Blantyre, Malawi. ${ }^{8}$ Department of Medicine, University of North Carolina School of Medicine, Chapel Hill, USA. 'Department of HIV and AIDS, Ministry of Health, Lilongwe, Malawi. ${ }^{10}$ Department of Public Health, University of Malawi, College of Medicine, School of Public Health and Family Medicine, Lilongwe, Malawi.

Received: 4 May 2017 Accepted: 30 June 2017

Published online: 11 July 2017

\section{References}

1. World Health Orgnization 2012. Programmatic Update: Use of antiretroviral drugs for treating pregnant women and preventing HIV infection in infants. Available from: http://www.who.int/hiv/pub/mtct/programmatic update2012/en/. Accessed 7 June 2017.

2. Center for Disease Control and Prevention (CDC). Impact of an innovative approach to prevent mother-to-child transmission of HIV-Malawi, July 2011September 2012. MMWR Morb Mortal Wkly Rep. 2013;62(8):148-51.

3. Haas A, Tenthani L, Msukwa M, Tal K, Jahn A, Gadabu O, et al. Retention in care during the first 3 years of antiretroviral therapy for women in Malawi's option B+ programme: an observational cohort study. Lancet HIV. 2016:3(4):e175-82

4. Schouten EJ, Jahn A, Midiani D, Makombe SD, Mnthambala A, Chirwa Z, et al. Prevention of mother-to-child transmission of HIV and the health-related millennium development goals: time for a public health approach. Lancet. 2011;378(9787):282-4.

5. Govindasamy D, Meghij J, Negussi EK, Baggaley RC, Ford N, Kranzer K. Interventions to improve or facilitate linkage to or retention in pre-ART (HIV) care and initiation of ART in low- and middle income settings - a systematic review. J Int AIDS Soc. 2014;17:19032

6. Hales S, Lesher-Trevino A, Ford N, Maher D, Ramsay A, Tran N. Reporting guidelines for implementation and operational research. Bull World Health Organ. 2016;94:58-64

7. Rosenberg NE, van Lettow M, Tweya H, Kapito-Tembo A, Man Bourdon C, Cataldo F, et al. Improving PMTCT uptake and retention services through novel approaches in peer-based family-supported Care in the Clinic and Community: a three-arm cluster randomized trial (PURE Malawi). J Acquir Immune Defic Syndr. 2014;67:S114-9.

8. Ministry of Health Malawi. Clinical Management of HIV in Children and Adults 2014. Ministry of Health, Malawi. Available at: http://www.emtct-iatt. org/wp-content/uploads/2015/09/Malawi-HIV-Guidelines-2014.pdf. Accessed 7 June 2017.

9. World Health Organization (2012). Male involvement in the prevention of mother-to-child transmission of HIV. Available at: http://apps.who.int/iris/ bitstream/10665/70917/3/9789241503679_eng.pdf. Accessed 7 June 2017.

10. Manjate Cuco RM, Munguambe K, Bique Osman N, Degomme O, Temmerman M, Sidat MM. Male partners involvement in preventionofmother-to-child HIV transmission in sub-Saharan Africa: a systematic review. Sahara J. 015(12):87-105. doi:10.1080/17290376.2015.1123643.

11. Takah NF, Kennedy ITR, Johnman C. Impact of approaches in improving male partner involvement in the prevention of mother-to-child transmission (PMTCT) of HIV on the uptake of PMTCT services in sub-Saharan Africa: a protocol of a systematic review and meta-analysis. BMJ Open. 2016;6:e012224. doi:10.1136/bmjopen-2016-012224.

12. Malawi Demographic and Health Survey 2010. Avaialble at: https://dhsprogram. com/pubs/pdf/FR247/FR247.pdf. Accessed 7 June 2017.

13. Phiri S, Tweya H, van Lettow M, Rosenberg NE, Trapence C, Kapito-Tembo A, et al. Impact of facility- and community-based peer support models on maternal uptake and retention in Malawi's option B+ HIV prevention of mother-to-child transmission program: a 3-arm cluster randomized controlled trial (PURE Malawi). J Acquir Immune Defic Syndr. 2017:75(Suppl 2):S140-8.

14. Cataldo F, Chiwaula L, Nkhata M, van Lettow M, Kasende F, Rosenberg NE, Tweya H, Sampathkumar V, Hosseinipour M, Schouten E, Kapito-Tembo A, Eliya M, Chimbwandira F, Phiri S. Exploring the Experiences of Women and Health Care Workers in the Context of PMTCT Option B Plus in Malawi. J Acquir Immune Defic Syndr. 2017;74:517-22. 